\title{
ICE MANTLE FORMATION IN THE ENVELOPES OF OH/IR STARS
}

\author{
S.B. CHARNLEY ${ }^{\dagger}$ \\ Space Science Division, NASA Ames Research Center, California, USA \\ and \\ R.G. SMITH \\ Dept. of Physics, University of New South Wales, Campbell, Australia
}

\begin{abstract}
We have computed ice column densities for a sample of O-rich late-type stars.
Water ice is observed in the outflows from several late-type stars (e.g. Smith et al. 1988) and it is of some observational and theoretical importance to understand how common ice mantles may be and how their formation depends upon the physical conditions in the envelope, such as the radial distributions of density and temperature. We have developed a simple theory of the gas-grain interaction in circumstellar shells (Jura \& Morris 1985; Charnley et al. 1992) and used it to compute ice column densities $\left(\mathrm{N}_{i c e}\right)$ and mantle thicknesses (d) for a sample of well-studied OH/IR stars (Herman \& Habing 1985). The models are most sensitive to the dust and gas mass loss rates, as well as to the inner radius of the envelope, $\mathrm{R}_{i}$, which is poorly determined. Representative results are presented in the Table below where $R_{i}$ was estimated according to the prescription of Herman et al. (1986).
\end{abstract}

\begin{tabular}{llllll} 
OBJECT & $\mathrm{R}_{i} / \mathrm{R}_{*}$ & $\begin{array}{l}\mathrm{R}_{i} \\
(\mathrm{~cm})\end{array}$ & $\begin{array}{l}\mathrm{R}_{\text {ice }} \\
(\mathrm{cm})\end{array}$ & $\begin{array}{l}\mathrm{N}_{i c e} \\
\left(\mathrm{~cm}^{-2}\right)\end{array}$ & $\begin{array}{l}\mathrm{d} \\
(\mu \mathrm{m})\end{array}$ \\
\hline & & & & & \\
$12.3-0.2$ & 7.2 & $5.8(+14)$ & $6.3(+16)$ & $1.4(+17)$ & $1.4(-02)$ \\
$12.8-1.9$ & 9.6 & $2.4(+14)$ & $2.3(+16)$ & $2.5(+15)$ & $2.8(-03)$ \\
$13.1+5.0$ & 8.4 & $4.3(+14)$ & $4.1(+16)$ & $3.0(+16)$ & $1.2(-02)$ \\
$16.1-0.3$ & 15.0 & $5.7(+13)$ & $4.3(+15)$ & $2.5(+16)$ & $1.7(-03)$ \\
$17.7-2.0$ & 10.8 & $1.3(+14)$ & $9.8(+15)$ & $1.6(+17)$ & $9.0(-03)$ \\
$20.2-0.1$ & 10.0 & $2.5(+14)$ & $2.4(+16)$ & $2.4(+16)$ & $5.2(-03)$ \\
$21.5+0.5$ & 5.9 & $1.7(+15)$ & $1.6(+17)$ & $8.6(+16)$ & $2.6(-02)$ \\
$26.4-1.9$ & 11.1 & $1.1(+14)$ & $1.0(+16)$ & $1.0(+16)$ & $5.9(-03)$ \\
$26.5+0.6$ & 8.9 & $2.5(+14)$ & $2.4(+16)$ & $3.9(+16)$ & $9.3(-03)$ \\
$30.1-0.7$ & 10.0 & $2.1(+14)$ & $1.6(+16)$ & $5.2(+16)$ & $4.8(-03)$ \\
$30.7+0.4$ & 6.8 & $7.5(+14)$ & $7.1(+16)$ & $4.3(+16)$ & $1.4(-02)$ \\
$32.0-0.5$ & 6.6 & $6.3(+14)$ & $6.0(+16)$ & $5.5(+16)$ & $9.3(-03)$ \\
$32.8-0.3$ & 6.1 & $1.1(+15)$ & $1.0(+17)$ & $9.0(+16)$ & $1.9(-02)$
\end{tabular}

\section{References}

Charnley, S.B., Smith, R.G. \& Brown, P.D. 1992, in preparation.

Herman, J. and Habing, H.J. 1985. Physics Reports, Vol. 124, No. 4, pp 255-314.

Herman, J., Burger, J.H. and Penninx, W.H. 1986, Astr.Ap., 167, 247.

Jura, M. and Morris, M. 1985, Ap.J., 292, 487.

Smith, R.G., Sellgren, K. and Tokunaga, A.T. 1988, Ap.J., 334, 209.

$\dagger$ NAS/NRC Resident Research Associate 